\title{
A new way of thinking about pain
}

\author{
John D Loeser*,1 \\ ${ }^{1}$ Departments of Neurological Surgery \& Anesthesiology \& Pain Medicine, University of Washington, Seattle, 98195 WA, USA \\ *Author for correspondence: Tel.: +1 206543 3570; Fax: +1 206543 8315; jdloeser@uw.edu
}

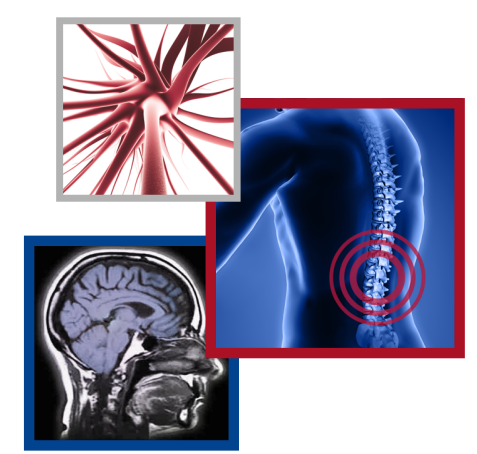

First draft submitted: 13 August 2018; Accepted for publication: 18 September 2018; Published online: 5 December 2018

Keywords: acute pain $\bullet$ central pain $\bullet$ chronic pain

For pain states that are traditionally classified as acute or chronic, I propose a different dichotomy - peripherally driven or centrally maintained. This is not an original concept by the current author, as it can be found in the first edition of Bonica's Management of Pain, published in 1953 [1]. In Bonica's original discussion, there is no mention of acute versus chronic pain. Instead, he writes of peripheral and central pains. As is so often the case, he was many years ahead of the rest of us in thinking about pain. The dichotomy of acute versus chronic became pre-eminent as the development of pain management services in the 1970s progressed - the need to identify the appropriate patients for referral to a pain center fostered this categorization. Only chronic pain patients were thought to need referral to multidisciplinary pain centers. The second edition of Bonica's Management of Pain, published in 1990, extensively discussed the differences between acute and chronic pain, and added mention of the fact that chronic pain could have major psychological factors [2]. As Bonica noted in both editions, we have rather good treatments for most peripherally driven pains, but much less efficacy with centrally maintained pains.

The idea that acute pains transition into chronic is without an evidential underpinning and, I believe, is an error in conceptualization [3]. There is no known physiologic or anatomic change that occurs at 3 or 6 months after onset of a painful condition. Utilizing duration as a critical factor may obscure the role of other factors such as affective state, coping strategies and past history in perpetuating chronic pain. Centrally maintained pains do have an onset and in that sense can be temporally 'acute'. A patient who sustains a stroke involving the spinothalamic system may develop contralateral hemibody pain at the time of the stroke. It may persist for the remainder of the patient's life. Should such a pain be called 'acute' for 90 days and thereafter be called 'chronic'? This pain is due to a lesion in the nervous system; it is centrally maintained from its onset. Peripherally driven pains may persist for long periods of time but that does not make them central pain states. Most patients with knee or hip joint replacement awaken from their anesthetic and complain of incisional pain but are amazed at the absence of their preoperative hip or knee pain. I believe that the terms, 'acute' and 'chronic' obfuscate the basis for a pain syndrome and should be abolished.

Some centrally driven pains are related to a clear-cut injury to the nervous system, which is manifested by loss of sensory or motor function. The clinical picture makes it certain that the ensuing pain is centrally generated even though the patient reports pain in a specific part of the body. The folly of pursuing pain relief in the painful part is demonstrated by the history of attempts to relieve the pains of postherpetic neuralgia by excising the painful region of the skin, often followed by peripheral neurectomy, dorsal rhizotomy, anterolateral cordotomy, thalamotomy and cortical resection - none of which provided long-term pain relief. The literature is large for these clear-cut pains associated with detectable nervous system dysfunction, but the underlying mechanism(s) remain hypothetical at best. In spite of the fact that the pain is always ascribed to a region of the body, the causative mechanism cannot be found where it hurts. This is also clearly demonstrated by phantom limb pain - the part that hurts is no longer connected to the body.

The acute-chronic dichotomy has played a significant role in the failure of infrahuman pain models to lead to successful pain treatment strategies. As clearly described by Yezierski and Hanson [4], the use of peripherally generated pain models in experimental animals has not yielded useful drugs or treatment strategies for centrally maintained pain states. It is much harder to study brain or spinal cord changes, but I believe this is where the action lies for centrally maintained painful states. The paucity of information about the mechanisms of central 
pains contrasts with the amazing increase in our understandings of nociception, which is the basis for peripherally generated pains.

In the past 25 years, increasing attention has been devoted to pain states that are not associated with a neurological deficit but are still presumed to be due to CNS dysfunction. This is often because of the presence of sensory processing abnormalities such as allodynia in the region of pain. In addition, no pathology has been located in the regions of pain. An increasing number of pain syndromes have been placed in this category. I would like to label such pains as 'mysterious central pains'. Such pains are ascribed to almost every organ system or region of the body and they have been responsible for many failed treatments aimed at the part that hurts. Some examples include:

- Noncardiac chest pain;

- Nonspecific gastritis;

- Nonspecific prostatitis;

- Migraine, other headaches;

- Fibromyalgia;

- Irritable bowel syndrome;

- Interstitial cystitis; and

- Failed back surgery syndrome (some).

Furthermore, I suggest that these mysterious central pain states, like fibromyalgia, for example, are best thought of as a nocebo (nonspecific) response to the internal and external events of life. We know that about one out of three of the favorable responses to treatment are placebo (or nonspecific) based; it is reasonable to predict that there are an equal number of nocebo responders. I suggest that such mysterious central pain patients are manifesting a nocebo response to messages coming from their bodies. We should stop trying to treat them with biomedically based therapies and shift to psychological strategies designed to reduce nonspecific treatment effects. Nocebo effects make use of a complex set of neural circuits in the CNS that modulate all perceptions. We know that conditioning and expectations are important for nocebos [5]. Consequently, psychological strategies can be used to modify them. An example of such a therapeutic approach is the Explaining Pain model of Moseley and Butler - changing the patient's understanding of biological processes can change the pain experience [6]. Voodoo shamans have been reported to induce vomiting, dizziness, headaches and even death - triggered through belief alone. Placebo and nocebo effects are associated with opposite responses of dopamine and endogenous opioid neurotransmission in a distributed network of regions. The brain areas involved in these phenomena form part of the circuit typically implicated in reward responses and motivated behavior [7].

Repeated surveys have found that approximately $75 \%$ of people will endorse the statement that they have chronic pain but that only $5 \%$ will seek healthcare, that is, become patients. Having pains is an age-related phenomenon that does not necessarily imply seeking healthcare. Long-term pain in the absence of a peripheral generator leading to becoming a patient indicates a nonspecific response to life's stressors, physical and emotional. This is why thorough studies of many chronic pain patients fail to find a 'cause' - usually meaning something wrong in the body. What is wrong is the way such people deal with the stressors of life in a nonspecific way, which is a nocebo response.

Dichotomizing the world of pain into acute versus chronic does not lead to improved understanding of this fundamental human experience. It is time that we abolished the use of such a temporal schism and more accurately identified pain syndromes as generated in the periphery or in the nervous system. Both patient care and research would be facilitated by such a conceptual change.

Financial \& competing interests disclosure

The authors have no relevant affiliations or financial involvement with any organization or entity with a financial interest in or financial conflict with the subject matter or materials discussed in the manuscript. This includes employment, consultancies, honoraria, stock ownership or options, expert testimony, grants or patents received or pending, or royalties.

No writing assistance was utilized in the production of this manuscript.

\section{References}

1. Bonica JJ. The Management of Pain. Lea \& Febiger, Philadelphia, USA (1953).

2. Bonica JJ. The Management of Pain. Lea \& Febiger, Philadelphia, USA (1990). 
3. Dunn KM, Croft P. Defining chronic pain by prognosis. In: From Acute to Chronic Back Pain, Hasenbring M, Rusu A, Turk DC (Eds). Oxford University Press, Oxford, UK, 21-40 (2012).

4. Yezierski RP, Hansson P. Inflammatory and neuropathic pain from bench to bedside: what went wrong? J. Pain 19(6), 571-588 (2018).

5. Blasini M, Corsi N, Klinger R, Colloca L. Nocebo and pain: an overview of the psychoneurobiological mechanisms. Pain Reports 2(2), (2017).

6. Moseley GL, Butler DS. Fifteen years of explaining pain: the past, present, and future. J. Pain 16(9), 807-813 (2015).

7. Scott DJ, Stohler CS, Egnatuk CM, Wang H, Koeppe RA, Zubieta JK. Placebo and nocebo effects are defined by opposite opioid and dopaminergic responses. Arch. Gen. Psychiatry 65(2), 220-231 (2008). 
